\title{
Nuclear Quadrupole Moments as a Microscopic Probe to Study the Motion of Atomic Tunneling Systems in Amorphous Solids
}

\author{
M. Bartkowiak,* M. Bazrafshan, C. Fischer, A. Fleischmann, and C. Enss \\ Kirchhoff-Institut für Physik, Universität Heidelberg, INF 227 D-69120 Heidelberg, Germany
}

(Received 6 February 2013; published 13 May 2013)

\begin{abstract}
The properties of amorphous solids below $1 \mathrm{~K}$ are dominated by atomic tunneling systems. A basic description is given by the standard tunneling model. Despite its success, the standard tunneling model still remains phenomenological and little is known about the microscopic nature of tunneling systems in amorphous solids. We present dielectric polarization echo experiments on partially deuterated amorphous glycerol. Nuclear quadrupoles, introduced by the deuteration, influence the echo amplitude in a characteristic way and allow us to draw for the first time detailed conclusions about the microscopic nature of the tunneling processes in amorphous glycerol.
\end{abstract}

DOI: 10.1103/PhysRevLett.110.205502

PACS numbers: 61.43.Fs, 77.22.- d

Localized atomic tunneling systems and their interaction with other excitations such as phonons govern the lowtemperature properties of amorphous solids. Their detailed understanding is of fundamental importance not only to describe glasses at low temperatures but also because of many implications for other experiments. For example, the enhanced dielectric loss of amorphous insulators and the generation of $1 / f$ noise $[1,2]$ in such systems degrade the performance in applications like kinetic inductance detectors [3] and flux-biased superconducting phase qubits $[4,5]$. On the other hand, atomic tunneling systems with long coherence times have been used themselves as computational qubits [6]. Very recently, the relaxation and dephasing of atomic tunneling systems have been studied in a Josephson junction [7] and the strain tuning of individual atomic tunneling systems has been detected via a superconducting qubit [8]. Despite this progress, the microscopic nature of atomic tunneling systems in amorphous solids is still unknown and remains a fundamental and unsolved question. So far, sophisticated computer simulations have been the only approach to obtain information on the structural configuration of tunneling systems in amorphous materials (for a review, see [9]). In the absence of a microscopic theory, the low-temperature properties of insulating glasses are generally described within the framework of the standard tunneling model [10,11] (for reviews, see $[12,13])$. In this phenomenological approach, a tunneling system is depicted by a particle with mass $m$ residing in a double well potential with an asymmetry energy $\Delta$ between the two wells and a tunneling matrix element $\Delta_{0}=$ $\hbar \omega \exp (-d \sqrt{2 m V} / \hbar)$, where $V$ denotes the barrier height and $d$ the distance between the two wells. The resulting energy splitting between the two lowest delocalized eigenstates is given by $E=\sqrt{\Delta^{2}+\Delta_{0}^{2}}$. In order to account for the broad variety of tunneling systems found in glasses, the density of states is assumed to be a slowly varying function of these parameters and can be written as

$$
P(E, q) d E d q=\frac{\bar{P}}{1-q^{2}} d E d q
$$

with $q=\Delta / E$. The lack of a microscopic description despite numerous experimental efforts is in part due to the fact that the low-temperature properties of glasses are almost universal and depend only very little on details of their composition. Recent dielectric polarization echo experiments have shown that the echo amplitude for certain glasses depends on magnetic fields and that the behavior is nonuniversal; i.e., different materials show different magnetic field dependencies [14-17]. Soon it was realized that this magnetic field dependence is caused by tunneling particles carrying a nuclear quadrupole moment [18]. Meanwhile elaborate theoretical models exist to describe the effect $[19,20]$. In the present work we show that the nonuniversal behavior found in the magnetic-field-dependent echo experiments can be used to obtain microscopic information on the tunneling motion in amorphous materials, because the nuclear quadrupole moments of tunneling particles can be considered as local probes.

Here we present first results on partially deuterated glycerol and show that this approach indeed leads to direct microscopic information. The dielectric echo experiments were performed on two differently deuterated glycerol samples, namely glycerol-d5 $\left(\mathrm{C}_{3} \mathrm{D}_{5}(\mathrm{OH})_{3}\right)$ and glycerold3 $\left(\mathrm{C}_{3} \mathrm{H}_{5}(\mathrm{OD})_{3}\right)$, in a dilution refrigerator at working temperatures of about $5 \mathrm{mK}$, using a reentrant microwave resonator. The sample in form of a drop was placed in the uniform electric field region between the center post and the bottom of the $\lambda / 4$-resonator. The disclike space filled with sample had an approximate diameter of $8 \mathrm{~mm}$ and a thickness of $0.3 \mathrm{~mm}$. The resonator has a ground mode frequency of about $1 \mathrm{GHz}$ (i.e., probing tunneling systems with an energy splitting of about $50 \mathrm{mK}$ ). Homogeneous magnetic fields up to about $200 \mathrm{mT}$ could be applied at the sample utilizing a homemade superconducting solenoid enclosing the resonator. We generated spontaneous 


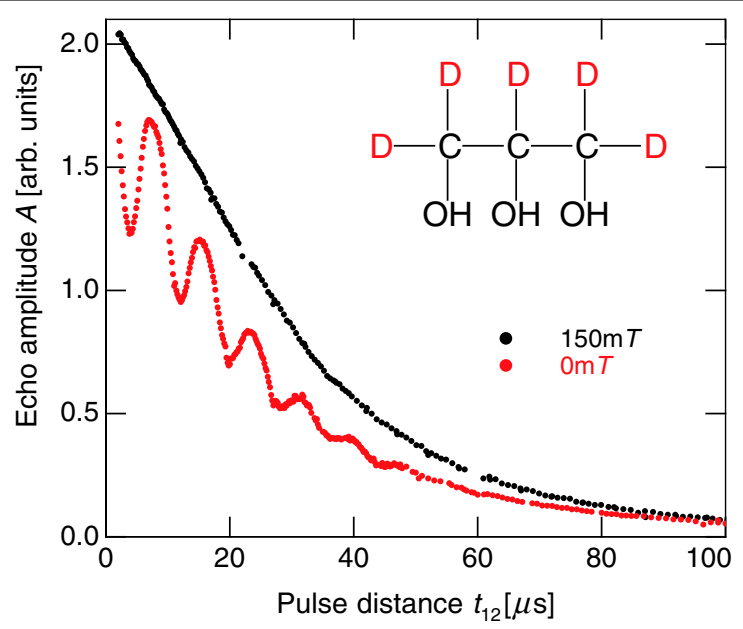

FIG. 1 (color online). Amplitude of two pulse polarization echoes generated in glycerol-d5 at $4 \mathrm{mK}$ as a function of the delay time $t_{12}$. The black points represent the amplitude of echoes taken in a magnetic field of $150 \mathrm{mT}$ and the red points, zero field measurements.

polarization echoes by applying two short rf pulses with a length of the order of several hundred nanoseconds resulting in nutation angles close to $\pi / 2$ and $\pi$, respectively.

Figures 1 and 2 show the amplitude of polarization echoes generated in glycerol-d5 and glycerol-d3 in zero magnetic field and in $150 \mathrm{mT}$ as a function of the delay time $t_{12}$ between the two excitation pulses. Clearly, the decay curves are very different with and without magnetic field for both samples. Whereas monotonic decays are observed for the measurements in $150 \mathrm{mT}$, pronounced oscillations are overlayed in case of the zero magnetic field measurements. A second observation is that the amplitude

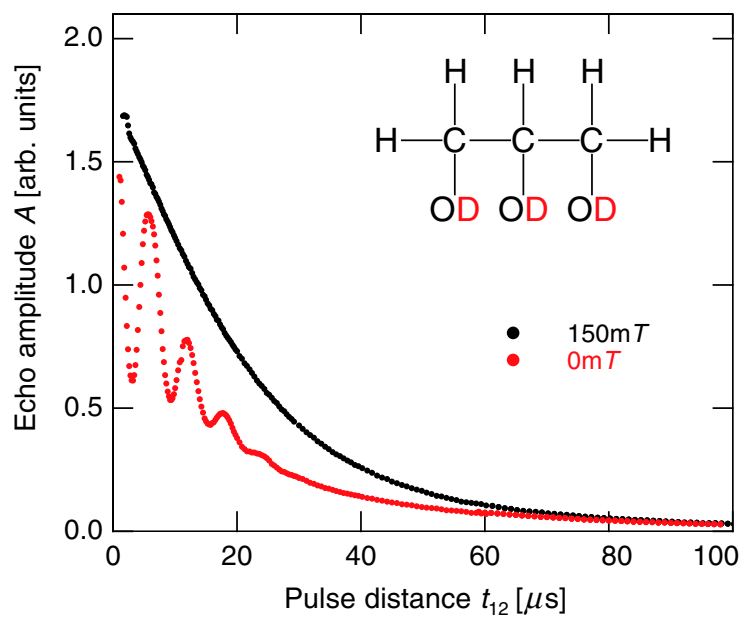

FIG. 2 (color online). Amplitude of two pulse polarization echoes generated in glycerol-d3 at $4 \mathrm{mK}$ as a function of the delay time $t_{12}$. The black points represent the amplitude of echoes taken in a magnetic field of $150 \mathrm{mT}$ and the red points, zero field measurements. of the data taken at zero field is somewhat smaller in the entire time window.

The monotonic decay reflects phase-disturbing processes due to spectral diffusion processes [21]. This has been studied in many glasses before and is not the focus of this work. Here we will concentrate on the overlaying oscillations, which originate from an interference effect within the level structure of the tunneling systems carrying a nuclear quadrupole moment. The presence of a nuclear quadrupole moment on the tunneling entity will yield a splitting of the two tunneling states into sublevels, which correspond to the eigenstates of the quadrupole [18]. For asymmetric tunneling systems $\Delta \neq 0$ with electric field gradients that are different in the two wells, transitions between the nuclear substates will be enforced. On the other hand, for perfectly symmetric tunneling systems $\Delta=0$, no transitions between the nuclear sublevels are induced by the tunneling motion.

In the following we will discuss this effect for glycerol-d5 in more detail, since for C-D bonds in general, the quadrupole Hamiltonian simplifies considerably. The field gradient is mainly defined by the C-D bond and shows almost perfect cylindrical symmetry about the bonding axis [22]. In glycerol, the quadrupole splitting is an intra-molecular property and is insensitive to changes in the surroundings of the molecule. However, the nuclei will experience different electric field gradients in the two wells when the tunneling motion includes a rotational component, since the relative orientation of the electric field gradient (determined by the intramolecular axis) will change with respect to the nuclear spin. Note that the spin lattice relaxation time for amorphous dielectrics at millikelvin temperatures is orders of magnitude longer than the time scale of the tunneling process [23]. As discussed above, a difference in electric field gradients of the two wells may lead to a finite transition probability between the nuclear substates. Since the transition probabilities depend on the magnitude of the asymmetry energy and the tunneling angle, they can be used to determine microscopic parameters of the tunneling systems contributing to the echo experiments. The transition probabilities can be retrieved from both the quantum beating of the echo amplitude in zero magnetic field and from magnetic-fielddependent measurements of the echo amplitude at different fixed delay times.

Let us first consider the case of zero magnetic field and glycerol-d5. As mentioned above, the level structure for deuterons in C-D bonds is particularly simple because of its uniaxial symmetry, and the quadrupole Hamiltonian can be represented by [24]

$$
\begin{aligned}
\hat{\mathcal{H}}_{Q}(\theta)= & \frac{e^{2} q Q}{4 I(2 I-1)}\left[\frac{1}{2}\left(3 \cos ^{2} \theta-1\right)\left(3 I_{z}^{2}-I(I+1)\right)\right. \\
& +\frac{3}{2} \cos \theta \sin \theta\left[I_{z}\left(I_{+}+I_{-}\right)+\left(I_{+}+I_{-}\right) I_{z}\right] \\
& \left.+\frac{3}{4} \sin ^{2} \theta\left(I_{+}^{2}+I_{-}^{2}\right)\right],
\end{aligned}
$$




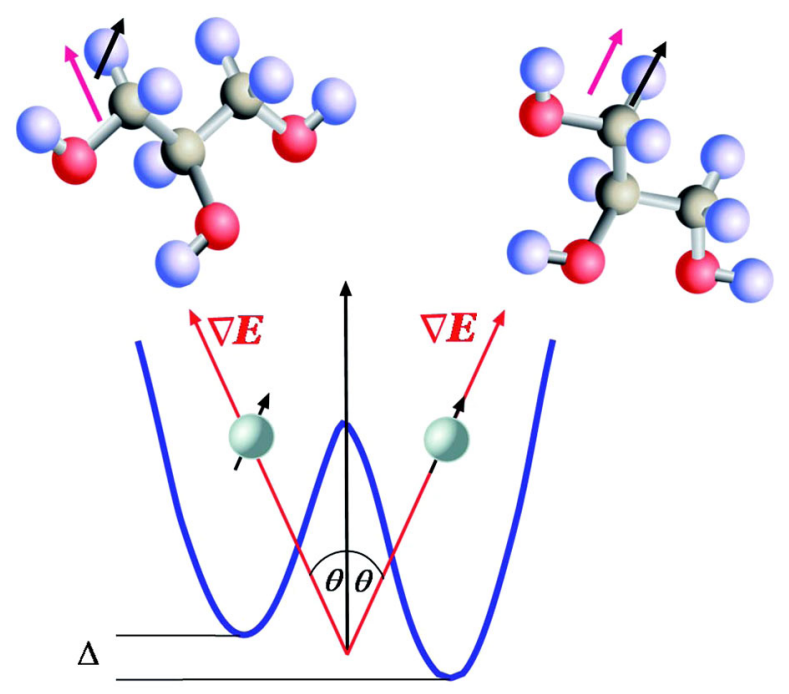

FIG. 3 (color online). Schematic of a deuterated glycerol molecule residing in an asymmetric double well potential. The two minima are reached by a rotational motion about an angle of $2 \theta$. For one deuteron the direction of the electric field gradient and the nuclear quadrupole moment are indicated by red and black arrows, respectively.

with the spin of a deuterium nucleus $I=1$. Here, $I_{z}$ is the $z$ component of the nuclear spin, and $\theta$ defines the angle between $I_{z}$ and the direction of the symmetry axis of the electric field gradient. We choose the quantization axis of $I_{z}$ such that the tilting angle $\theta$ takes the values $\theta$ and $-\theta$ in the left and the right well, respectively, as depicted in Fig. 3. In zero magnetic field, the levels $I_{z}= \pm 1$ are degenerate and thus the nuclear spin multiplet reduces to a doublet, whose levels are separated by the nuclear quadrupole energy $E_{Q}$.

In the polarization echo experiments discussed here, the first short pulse drives the ensemble coherently into a mixed state, leading to an oscillating macroscopic dipole moment. Since the energy splitting $E$ is broadly distributed in glassy systems, this oscillating dipole moment vanishes rapidly. The mixed state is a superposition of all eigenstates. As it evolves freely in time, each eigenstate accumulates a phase according to is energy eigenvalue. A second pulse at $t=t_{12}$ mixes the states again and induces a time reversal of the phase development, leading to a constructive interference at $t=2 t_{12}$ of eigenstates independent of their energies, whose relative phase accumulation is zero or a multiple of $2 \pi$. The occurrence of this macroscopic polarization is called polarization echo. The amplitude of such an echo for a multilevel system has been calculated independently by Würger [19] and Parshin [20]. For glycerol-d5 with five C-D bonds, one finds for the amplitude

$$
A_{\mathrm{d} 5}=A_{0}\left(t_{12}\right)\left[1-\frac{16}{3}\left(\frac{\Delta}{E} \sin (2 \theta)\right)^{2} \sin ^{4}\left(\frac{E_{Q} t_{12}}{2}\right)\right]^{5},
$$

where $A_{0}\left(t_{12}\right)$ is the echo amplitude for a two-level system including the decay, described by spectral diffusion. This equation can be used to evaluate the zero field data of glycerol-d5. From the period of the oscillations we derive a quadrupole frequency $E_{Q} / h$ of about $127 \mathrm{kHz}$. This agrees well with the value obtained by Schnauss et al. using ${ }^{2} \mathrm{H}-\mathrm{NMR}$ [25]. Integrating over the tunneling model distribution (1), a tunneling angle $2 \theta$ of about $17^{\circ}$ corresponds to a quantum beating of $35 \%$. If, however, the tunneling motion involves more than one molecule, the number of quadrupoles contributing will increase and the tunneling angle will be smaller. With the present set of data this cannot be excluded, and therefore the tunneling angle of $17^{\circ}$ has to be seen as an upper bound. Interestingly, preferred libration angles of this order of magnitude have been observed in NMR experiments near the glass transition temperature [25].

As shown in Fig. 1, the quantum beating vanishes at long delay times. In part this is due to a distribution of quadrupole frequencies. However, this distribution for C-D bonds in glycerol is rather narrow and can only account for a small portion of this effect [25]. The main reason for the fading of the beating amplitude is that tunneling systems with large asymmetries $\Delta$ are more strongly affected by phase disturbing processes [21] and therefore have shorter decay times. At long times symmetric tunneling systems stay coherent and govern the echo amplitude entirely, but do not contribute to the beating effect according to Eq. (3).

Let us now turn to the results for glycerol-d3. Here the electric filed gradient does not have perfect cylindrical symmetry, and thus the degeneracy of the $I_{z}=1$ and $I_{z}=-1$ states is lifted. This additional splitting influences the interference of the eigenstates and one expects a somewhat larger modulation amplitude for glycerol-d3. The fact that the deuterons in glycerol-d3 are involved the network forming hydrogen bonds makes modeling of the influence of the quadrupols of glycerol-d 3 on the echo amplitude much more involving than in case of glycerol-d5. Therefore, a detailed calculation of the expected modulation of the echo amplitude will not be discussed here. As shown in Fig. 2 the beating amplitude is about $60 \%$. Furthermore, the beat frequency is roughly $150 \mathrm{kHz}$, which is significantly larger than in case of glycerol-d5. Again this value is in very good agreement with quadrupole frequency of $150 \mathrm{kHz}$ obtained in previous NMR measurements [25]. The clear differences in the zero field data of glycerol-d5 and glycerol-d3 prove that microscopic information can be obtained utilizing the quadrupole effect.

Using the parameters for glycerol-d5, we can also describe the magnetic-field dependence of the echo amplitude. Corresponding data taken at $t_{12}=4 \mu \mathrm{s}$ and $T=12 \mathrm{mK}$ are shown in Fig. 4. In finite magnetic fields, the Zeeman effect leads to a shift of the energy levels of the nuclear substates and lifts the degeneracy $I_{z}= \pm 1$. This changes 


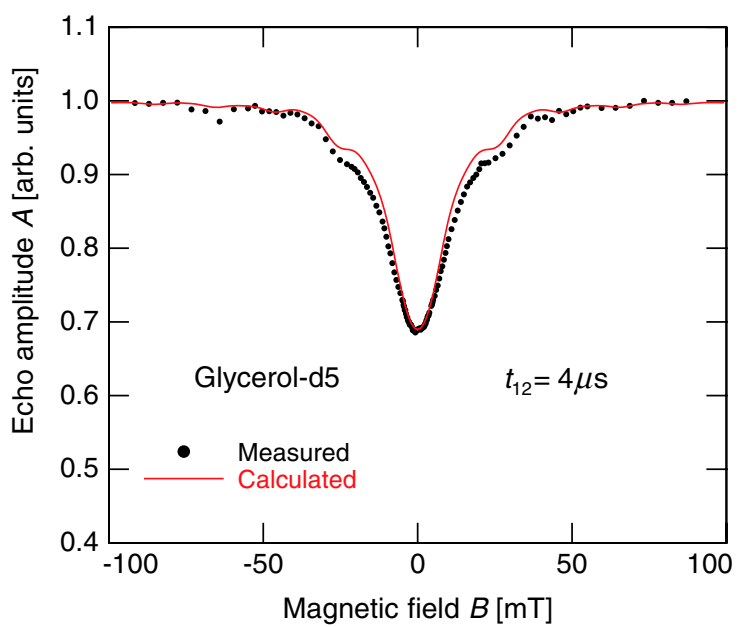

FIG. 4 (color online). Amplitude of polarization echoes generated in glycerol-d5 at $12 \mathrm{mK}$ and a delay time of $4 \mu \mathrm{s}$ as a function of the applied magnetic field. The solid line represents a numerical calculation based on a microscopic model of the tunneling motion.

the beat frequency and results in additional interference terms. However, in high fields the Zeeman interaction does not yield a mixing of multiplet states since this would correspond to a spin flip. The delay time at which the experimental data are taken (Fig. 4) corresponds roughly to the first minimum in the echo amplitude at zero magnetic field where destructive interference is maximum. The field induced change of the quantum beating frequency will drive the system out of this minimum position even if the lifting of the degeneracy introduces more interference terms. The echo amplitude is increasing monotonically and saturates above about $70 \mathrm{mT}$. At these fields the Zeeman energy has exceeded the quadrupole energy twice and suppresses the mixing of the nuclear substates completely, and the simple two-level system behavior is recovered. The small features (for example at about $40 \mathrm{mT}$ ) are due to level crossings within the multiplet. To model the field dependence, we have solved the problem numerically. First, we obtained the eigenvectors and eigenvalues of the full Hamiltonian, describing the tunneling an the nuclear spin states. Then, we calculated the matrix elements for transitions between the tunneling multiplets. To do so, the Hamiltonian describing the action of the rf pulses was applied to the eigenvectors. Using Eq. (15) in [20] the echo amplitude has been computed. Also the distribution of the parameters $\Delta$ and $\lambda$ according to the tunneling model were taken into account. In this calculation we further assumed that one molecule is tunneling about a certain angle $2 \theta$. As mentioned before, the orientation of the molecules with respect to the external field is completely arbitrary for each tunneling system. We accounted for this by distributing the orientation of the axis rotation of the tunneling motion evenly over the full solid angle $4 \pi$. Under these assumptions the best fit to the magnetic field dependence of the echo amplitude is obtained by using again a tunneling angle $2 \theta$ of about $17^{\circ}$. This means that the delay time dependence at zero magnetic field and the magnetic field dependence can be described reasonably well with the same set of parameters.

In conclusion, we have performed two pulse polarization echo experiments on partially deuterated glycerol as a function of the delay time and as a function of the applied magnetic field. Our results show that the echo amplitude depends on the deuteration of the glycerol and allow us to obtain for the first time experimental information on the microscopic nature of the atomic tunneling systems in amorphous materials. We believe that this is a new method to study systematically the microscopic nature of tunneling systems in glasses, which has been an unsolved problem for many decades.

We would like to thank U. Haeberlen, S. Hunklinger, M. v. Schickfus, G. Ruyters, A. Reiser, and H. Zimmermann for their support and many fruitful discussions. Part of this work is supported by the European Community Research Infrastructures under the FP7 Capacities Specific Programme, MICROKELVIN Project No. 228464.

*Present address: Paul Scherrer Institut, CH-5232 Villigen, Switzerland.

[1] A. Shnirman, G. Schön, I. Martin, and Y. Makhlin, Phys. Rev. Lett. 94, 127002 (2005).

[2] L. C. Ku and C. C. Yu, Phys. Rev. B 72, 024526 (2005).

[3] R. Barends, H. L. Hortensius, T. Zijlstra, J. J. A. Baselmans, S. J. C. Yates, J. R. Gao, and T. M. Klapwijk, Appl. Phys. Lett. 92, 223502 (2008).

[4] J.M. Martinis et al., Phys. Rev. Lett. 95, 210503 (2005).

[5] R. W. Simmonds, M.S. Allman, F. Altomare, K. Cicak, K. D. Osborn, J. A. Park, M. Sillanpää, A. Sirois, J. A. Strong, and J. D. Whittaker, Quantum Inf. Process. 8, 117 (2009).

[6] G. Sun, X. Wen, B. Mao, J. Chen, Y. Yu, P. Wu, and S. Han, Nat. Commun. 1, 51 (2010).

[7] Y. Shalibo, Y. Rofe, D. Shwa, F. Zeides, M. Neeley, J. M. Martinis, and N. Katz, Phys. Rev. Lett. 105, 177001 (2010).

[8] G. J. Grabovskij, T. Peichl, J. Lisenfeld, G. Weiss, and A. V. Ustinov, Science 338, 232 (2012).

[9] A. Heuer, in Tunneling Systems in Amorphous and Crystalline Solids, edited by P. Esquinazi (Springer, Berlin, 1998), p. 459.

[10] P. W. Anderson, B. I. Halperin, and C. M. Varma, Philos. Mag. 25, 1 (1972).

[11] W. A. Phillips, J. Low Temp. Phys. 7, 351 (1972).

[12] Tunneling Systems in Amorphous and Crystalline Solids, edited by P. Esquinazi (Springer, Berlin, 1998).

[13] S. Hunklinger and C. Enss, in Insulating and Semiconducting Glasses, edited by P. Boolchand, Series 
of Directions in Condensed Matter Physics Vol. 17 (World Scientific, Singapore, 2000), p. 499.

[14] S. Ludwig, C. Enss, P. Strehlow, and S. Hunklinger, Phys. Rev. Lett. 88, 075501 (2002).

[15] C. Enss and S. Ludwig, Phys. Rev. Lett. 89, 075501 (2002).

[16] S. Ludwig, P. Nagel, S. Hunklinger, and C. Enss, J. Low Temp. Phys. 131, 89 (2003).

[17] P. Nagel, A. Fleischmann, S. Hunklinger, and C. Enss, Phys. Rev. Lett. 92, 245511 (2004).

[18] A. Würger, A. Fleischmann, and C. Enss, Phys. Rev. Lett. 89, 237601 (2002).
[19] A. Würger, J. Low Temp. Phys. 137, 143 (2004).

[20] D. A. Parshin, J. Low Temp. Phys. 137, 233 (2004).

[21] J. L. Black and B. I. Halperin, Phys. Rev. B 16, 2879 (1977).

[22] F. Fujara, W. Petry, R. M. Diehl, W. Schnauss, and H. Sillescu, Europhys. Lett. 14, 563 (1991).

[23] J. Szeftel and H. Alloul, Phys. Rev. Lett. 34, 657 (1975); 42, 1691 (1979).

[24] A. Abragam, Principles of Nuclear Magnetism (Oxford University, New York, 1961).

[25] W. Schnauss, F. Fujara, and H. Sillescu, J. Chem. Phys. 97, 1378 (1992). 